\title{
Autoreactive B cells evade BAFF blockade in a mouse model of SLE
}

$\mathrm{H}$ ow does therapeutic targeting of B cells-with agents such as belimumab-work in systemic lupus erythematosus (SLE)? Much remains unknown about B-cell biology in SLE; whether pathogenic autoantibody depletion (the initial rationale for targeting these cells) is actually the effective mechanism is far from clear. Anne Davidson and colleagues at the Feinstein Institute for Medical Research, New York, and the Hebrew University in Jerusalem now add new data. Their findings, in mice, confirm that not all germline-encoded autoreactive $B$ cells are eliminated by blockade of BAFF (B-cell activating factor, also known as TNF ligand superfamily member 13b), the target of belimumab.

The researchers introduced a transgene encoding the heavy chain of an anti-DNA antibody, D42, into autoimmune-prone mice, and analyzed B-cell populations and antibody expression. The animals expressed D42 in conjunction with a particular light chain, VkRF; this pairing produces high-affinity anti-DNA activity. "We were lucky with our results," says Davidson, "because we found that there was a marked alteration in selection of both the naive and germinal center (GC) repertoires in the SLE-prone NZB/W D42 mouse, allowing us to study both stages of
B-cell selection." She continues, "although most B cells using VkRF were deleted after the immature bone marrow stage, the few remaining cells were positively selected in the GCs."

BAFF inhibition caused the loss of 2 in 3 transgenic $B$ cells at the transitional stage of maturity. Nonetheless, as Davidson explains, "we still observed strong positive selection of the VkRF light chain in the GCs, and the generation of a serum IgG anti-DNA response with only a short delay compared with controls." Thus, some high-affinity germline-encoded autoreactive B cells escape deletion and are expanded in GCs in autoimmune-prone mice, despite BAFF inhibition.

"The methodology is very sound, indeed elegant," opines David Pisetsky of Duke University, North Carolina, an expert in SLE and B-cell biology. The use of a transgene system and autoimmune mice has drawbacks, he cautions, but the models are nevertheless "very informative".

"I think the important findings relate to the striking emergence of the antiDNA antibody specificity in spontaneous autoimmunity, and the limited effects of BAFF inhibition on this expression," continues Pisetsky. "Once again, these findings emphasize the importance of antigen selection in the anti-DNA response in lupus, and the checkpoints that may be defective."

The effect of BAFF inhibition on selection of autoreactive B cells generated de novo by somatic mutation cannot be studied using this model, and Davidson and colleagues are developing an alternative experimental system. As BAFF affects other immune cell types, "we are also determining whether other mechanisms could account for the therapeutic effects of BAFF inhibition," Davidson adds.

"Perhaps with established disease, blocking BAFF has only a limited ability to change autoantibody production," muses Pisetsky. Studies in humans will be crucial, he adds, "to understand the relationship of serology to clinical disease activity and drug efficacy."

\section{Emma Leah}

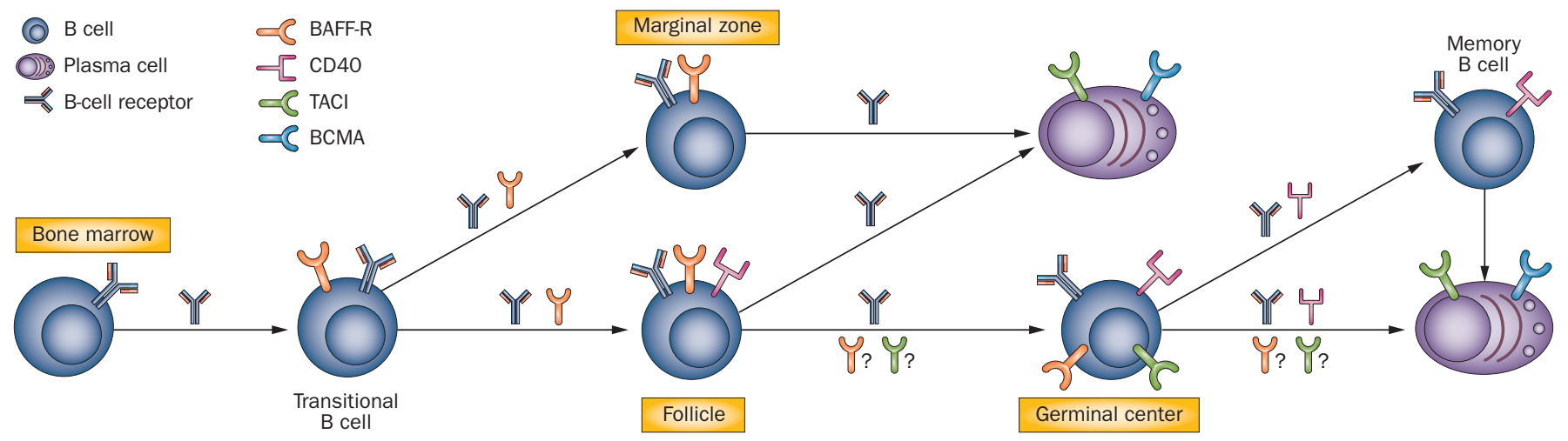

Receptor participation and T-cell help (in the form of CD40 ligation) in B-cell selection. BAFF-R contributes to the stringency of selection of the naive repertoire by interacting with B-cell receptor signalng pathways. CD40 ligation contributes to antigen-driven B-cell selection by facilitating survival and affinity maturation of B cells in the germinal center. TACl and BCMA are important mediators of plasma cell survival. Data from transgenic mice suggest that germinal center entry and expansion of high-affinity germline-encoded anti-DNA antibodies is not prevented by BAFF blockade. Abbreviations: BAFF, B-cell activating factor; BCMA, B-cell maturation factor; TACI, transmembrane activator and CAML interactor. 